\title{
Driving assembly far from equilibrium
}

1. Design principles for nonequilibrium self assembly

Authors: M. Nguyen and S. Vaikuntanathan

Proc. Natl. Acad. Sci., 113, 14231-14236 (2016)

2. Dissipation induced transitions in elastic strings

Authors: M. Nguyen and S. Vaikuntanathan

arXiv:1803.04368

Recommended with a Commentary by Michael F. Hagan, Martin
Fisher School of Physics, Brandeis University, Waltham, MA, USA

Nonequilibrium assembly and disassembly underlie key biological functions, such as growth, movement, and the dramatic morphological transformations that occur during organismal development. These processes are driven and regulated by energy dissipation, e.g. through nucleotide triphosphate hydrolysis. Energy consumption frees the organism from the rigid constraints of thermodynamic equilibrium, but also poses a significant challenge for researchers hoping to understand biological assembly or design synthetic systems with similar capabilities. While statistical mechanics can, in principle, predict equilibrium distributions of self-assembly reactions from microscopic interactions and dynamics, there is no analogous theory for nonequilibrium steady-states and the fluctuations around them. Despite recent progress through techniques such as computer simulations, large deviation theory, and stochastic thermodynamics (e.g. [1-11]), we are far from achieving a 'grand challenge' of nonequilibrium statistical mechanics: predicting the emergent products and functionalities of nonequilibrium assembly, or more ambitiously designing an assembly process to achieve a desired function.

This commentary will focus on a recent advance in nonequilibrium assembly described in two papers by Nguyen and Vaikuntanathan [7,12]. In [7], by using stochastic thermodynamics to calculate the entropy change during assembly and invoking the second law of thermodynamics, they obtained a bound on the nonequilibrium driving force required to perturb the ensemble of assembly configurations from its equilibrium distribution:

$$
\delta \mu-\left\langle\epsilon_{\text {diss }}\right\rangle \geq 0
$$

with $\delta \mu$ the nonequilibrium driving force and $\left\langle\epsilon_{\text {diss }}\right\rangle$ as the distance between the ensemble of assembly configurations and the equilibrium distribution. The quantity $\left\langle\epsilon_{\text {diss }}\right\rangle$ can be calculated, for example, as the difference between the equilibrium Hamiltonian for the system $E^{\mathrm{eq}}$ and an effective Hamiltonian $E^{\mathrm{eff}}$ that would lead to the observed configurational distribution (see below). They applied Eq. (1) to estimate compositional fluctuations in growing binary lattice systems [7]. 
Now, Nguyen and Vaikuntanathan [12] show that this result can also be applied to assembly morphologies. Focusing on a minimal model for assembly of an elastic membrane, they estimate the driving required to trigger morphology transitions. Their model is a semiflexible bead-spring ring polymer in two dimensions, which undergoes selfassembly by exchanging particles with a bath at constant chemical potential $\mu$ (Fig. 1). By simulating the dynamics with kinetic Monte Carlo, they find that there is a 'coexistence' value of the chemical potential, $\mu=\mu_{\text {coex }}$, for which the ring fluctuates around a constant size and is approximately circular. For larger chemical potential values the ring grows, with a rate that increases with $\delta \mu=\mu-\mu_{\text {coex }}$. Thus, $\delta \mu / k_{\mathrm{B}} T$, with $k_{\mathrm{B}} T$ the thermal energy, measures the strength of nonequilibrium driving. Above a threshold value of $\delta \mu / k_{\mathrm{B}} T$, the ring undergoes a buckling instability, exhibiting large dynamic 'spikes' (Fig. 1).

The key challenge for applying Eq. (1) to assembly morphology is that one must relate $\epsilon_{\text {diss }}$ to structural changes. To this end, Nguyen and Vaikuntanathan measure the fluctuations in particle positions from the mean ring radius, $\hat{h}$. At equilibrium the fluctuation spectrum can be described by assuming equipartition of energy among the modes of the standard continuum elastic Hamiltonian:

$$
E_{\mathrm{eq}}=\int\left\{\frac{\gamma}{2}(\nabla \hat{h})^{2}+\frac{\kappa}{2}(\Delta \hat{h})^{2}\right\} d x
$$

with $\gamma$ and $\kappa$ as the surface tension and bending modulus. Interestingly, although the ring undergoes continual growth for $\delta \mu>0$, Nguyen and Vaikuntanathan find that the fluctuation spectrum is still well described by equipartion and Eq. (2), but with renormalized values of the apparent moduli, $\sigma^{\text {eff }}$ and $\kappa^{\text {eff }}$. In particular, the effective surface tension decreases with increasing $\delta \mu$; below a critical value $\sigma_{\mathrm{c}}$ the ring undergoes the buckling instability. Importantly, although the effective surface tension and bending modulus $\sigma^{\text {eff }}$ and $\kappa^{\text {eff }}$ are not thermodynamic quantities, they are independent of the initial ring size or duration of measurement. Thus, Nguyen and Vaikuntanathan parameterize the ensemble of ring configurations by an 'effective energy landscape', obtained by substituting $\sigma^{\text {eff }}$ and $\kappa^{\text {eff }}$ into Eq. (1). The difference between the equilibrium and effective energy landscapes provides a measure for how much the ring morphology distribution deviates from equilibrium, $\left\langle\epsilon_{\text {diss }}\right\rangle$.

With this measure of distance from equilibrium in hand, Nguyen and Vaikuntanathan 
use Eq. (1) (and a tighter bound obtained by relating entropy production rates to current fluctuations) to relate the nonequilibrium driving to morphology changes. In particular, they show that the bounds estimate the renormalized surface tension with reasonable accuracy, even far from equilibrium.

Although the simulated system is (by design) a toy model, the results have fundamental implications for processes involving dynamically reconfiguring filaments and membranes, which play key functional roles in biology. More broadly, their results demonstrate that constraints on nonequilibrium driving predicted by stochastic thermodynamics can be directly related to material morphology transitions, thus establishing design principles for synthetic systems with functionalities similar to those found in living organisms.

The study also inspires open questions. The macroscale emergent behaviors of a nonequilibrium system depend on how it dissipates the energy injected by driving; i.e., which collective modes are most strongly excited [13]. The bound on magnitude of deviation from equilibrium (Eq. (1)) is thermodynamic in nature and independent of kinetic details. However, it does not constrain which modes are excited, and thus leaves the form of emergent behaviors unspecified. For example, Nguyen and Vaikuntanathan's simulations on the ring polymer show that energy preferentially dissipates through excess interfacial area rather than bending (since the renormalization of $\sigma^{\text {eff }}$ is much stronger than that of $\kappa^{\text {eff }}$ ). Would this result change if additional configurational relaxation moves are introduced to the Monte Carlo move set, or the assemblies have additional internal degrees of freedom? Conversely, how do we design dynamics and internal modes to achieve a desired morphology distribution? These questions, which require predicting how energy partitions among structural modes, return us to the grand challenge mentioned above. While these articles have not solved this challenge, they provide a stimulating step along this direction.

\section{References}

[1] T. R. Gingrich, J. M. Horowitz, N. Perunov, and J. L. England. Dissipation bounds all steady-state current fluctuations. Phys Rev Lett, 116(12):120601, 2016.

[2] U. Seifert. Stochastic thermodynamics, fluctuation theorems and molecular machines. Rep Prog Phys, 75(12):126001, 2012.

[3] U. Seifert. Stochastic thermodynamics: principles and perspectives. The European Physical Journal B, 64(3-4):423-431, 2008.

[4] Hugo Touchette. A basic introduction to large deviations: Theory, applications, simulations. arXiv:1106.4146, 2011.

[5] R. Marsland and J. L. England. Active regeneration unites high- and low-temperature features in cooperative self-assembly. Phys Rev E, 98(2-1):022411, 2018.

[6] Lishibanya Mohapatra, Bruce L Goode, Predrag Jelenkovic, Rob Phillips, and Jane Kondev. Design Principles of Length Control of Cytoskeletal Structures. Annu. Rev. Biophys., 45(April):85-116, 2016. 
[7] M. Nguyen and S. Vaikuntanathan. Design principles for nonequilibrium self-assembly. Proc Natl Acad Sci U S A, 113(50):14231-14236, 2016.

[8] R. K. P. Zia and B. Schmittmann. Probability currents as principal characteristics in the statistical mechanics of non-equilibrium steady states. Journal of Statistical Mechanics: Theory and Experiment, 2007(07):P07012-P07012, 2007.

[9] Shin-ichi Sasa and Hal Tasaki. Steady state thermodynamics. Journal of Statistical Physics, 125(1):125-224, 2006.

[10] J. R. Green, A. B. Costa, B. A. Grzybowski, and I. Szleifer. Relationship between dynamical entropy and energy dissipation far from thermodynamic equilibrium. Proceedings of the National Academy of Sciences of the United States of America, 110(41):16339-16343, 2013.

[11] Robert L. Jack. Large deviations in models of growing clusters with symmetry-breaking transitions. Physical Review E, 100(1), 2019.

[12] Michael Nguyen and Suriyanarayanan Vaikuntanathan. Dissipation induced transitions in two dimensional elastic membranes. arXiv:1803.04368, 2018.

[13] A. Joshi, E. Putzig, A. Baskaran, and M. F. Hagan. The interplay between activity and filament flexibility determines the emergent properties of active nematics. Soft Matter, 15(1):94-101, 2018. 